\title{
Dairy Consumption in Relation to Hypertension Among a Large Population of University Students: The MEPHASOUS Study
}

This article was published in the following Dove Press journal: Diabetes, Metabolic Syndrome and Obesity: Targets and Therapy

\author{
Masoume Mansouri, ' \\ Naseh Pahlavani, (iD) ${ }^{2}$ \\ Farshad Sharifi, ${ }^{3}$ \\ Mehdi Varmaghani, ${ }^{4}$ \\ Azad Shokri, (iD ${ }^{5}$ Hamid Yaghubi, ${ }^{6}$ \\ Omid Asbaghi, (iD) ${ }^{7}$ \\ Abasali Keshtkar, (iD) \\ Yousef Moghadas Tabrizi, \\ Omid Sadeghi ${ }^{10,11}$
}

'Student Health Services, Students' Health and Consultation Center, Tarbiat Modares

University, Tehran, Iran; ${ }^{2}$ Student Research

Committee, Faculty of Medicine, Mashhad

University of Medical Sciences, Mashhad, Iran;

${ }^{3}$ Elderly Health Research Center,

Endocrinology and Metabolism Research

Institute, Tehran University, Tehran, Iran; ${ }^{4}$ Social

Determinants of Health Research Center,

Mashhad University of Medical Sciences,

Mashhad, Iran; ${ }^{5}$ Social Determinants of Health

Research Center, Research Institute for Health

Development, Kurdistan University of Medical

Sciences, Sanandaj, Iran; ${ }^{6}$ Department of

Psychology, Shahed University, Tehran, Iran;

${ }^{7}$ Student Research Committee, Lorestan

University of Medical Sciences, Khorramabad,

Iran; ${ }^{8}$ Department of Health Sciences Education

Development, School of Public Health, Tehran

University of Medical Sciences, Tehran, Iran;

${ }^{9}$ Department of Health and Sport Medicine,

Faculty of Physical Education and Sport Science,

University of Tehran, Tehran, Iran; ${ }^{10}$ Students'

Scientific Research Center, Tehran University of Medical Sciences, Tehran, Iran; ' 'Department of Community Nutrition, School of Nutritional Sciences and Dietetics, Tehran University of Medical Sciences, Tehran, Iran

Correspondence: Omid Sadeghi

Department of Community Nutrition,

School of Nutritional Sciences and Dietetics,

Tehran University of Medical Sciences, P.o.

Box 14I55-61 I7, Tehran, Iran

Tel +98-2I-88955805

Fax +98-2I-8898486

Email osadeghi@razi.tums.ac.ir
Background: Hypertension is a chronic condition that its prevalence is increasing at an alarming rate. Findings on the association between dairy consumption and hypertension are conflicting and few data are available in the Middle East.

Aim: To assess the association between dairy consumption and hypertension among a large population of university students.

Materials and Methods: The current study was performed in the framework of the Mental and Physical Health Assessment of University Student (MEPHASOUS) project. Overall, 67,011 university students with complete information were included in the statistical analysis. To assess dairy consumption, a self-administered dietary habits questionnaire was employed. Blood pressure was measured using a standard protocol. The systolic/diastolic blood pressure of $\geq 140 / 90 \mathrm{mmHg}$ was considered hypertension.

Results: Hypertension was prevalent among $6.9 \%$ of students. A significant inverse association was found between dairy consumption and the odds of hypertension; such that after taking potential confounders into account, individuals in the highest levels of dairy consumption had $85 \%$ lower odds for having hypertension compared with those in the lowest levels (odds ratio (OR): $0.15,95 \%$ confidence interval (CI): 0.13-0.18). Such an inverse association was also seen among males (OR: 0.14, 95\% CI: $0.11-0.18)$ and females (OR: 0.16, 95\% CI: $0.12-0.21$ ), normal-weight students (OR: $0.15,95 \%$ CI: $0.12-0.18$ ) and those with overweight or obesity (OR: $0.15,95 \% \mathrm{CI}: 0.11-0.21$ ), and individuals with (OR: 0.13 , 95\% CI: 0.11-0.16) and without (OR: 0.24, 95\% CI: $0.17-0.35$ ) family history of hypertension.

Conclusion: Our results support the previous findings on the inverse association between dairy consumption and hypertension among university students.

Keywords: diet, hypertension, blood pressure, students, dairy

\section{Introduction}

Hypertension, as the blood pressure (BP) of $\geq 140 / 90 \mathrm{mmHg}$, is one of the major public health concerns worldwide and associated with increased risk of unfavorable cardiovascular diseases (CVDs) and mortality. ${ }^{1,2}$ In 2000, hypertension was prevalent among $26 \%$ of adults worldwide, and its prevalence is expected to increase to $29 \%$ in $2025 .^{3}$ Overall, it seems that promoting appropriate strategies for the prevention of hypertension is essential.

Several approaches including lifestyle modifications, particularly dietary management, have been proposed for the prevention of hypertension. Nutritional 
guidelines have recommended an energy-restricted diet with a high amount of fruits, vegetables, whole grains and with a reduced content of salt, saturated fatty acids, and dietary cholesterol. ${ }^{4,5}$ These recommendations have been purposed to reach a healthy body weight and to provide a balance between beneficial (potassium and calcium) and harmful (sodium) minerals for blood pressure. ${ }^{6}$ Dairy products contain a well-balanced composition of micronutrients that may help for the prevention of hypertension. ${ }^{6,7}$ In addition, these products are rich in bioactive tripeptides which provide an inhibitory effect on angiotensinconverting enzyme (ACE). ${ }^{8-10}$ Nevertheless, data on the association between dairy consumption and risk of hypertension are conflicting. In the Framingham Heart Study, which was performed on 2636 adults, Wang et al reported that dairy consumption, as part of a nutritious and energybalanced diet, was inversely associated with BP and incident hypertension. ${ }^{11}$ Conversely, in a Mendelian randomization study on 197,332 participants, genetically determined dairy consumption (using lactase persistence gene) was not associated with systolic blood pressure and risk of hypertension. ${ }^{12}$ However, due to the different lactose content of dairy products, interpreting Mendelian results is difficult. The observed inconsistencies on the association between dairy consumption and hypertension might be explained by different populations of previous studies, different processing of dairy products in different cultures, and controlling for different confounders in previous studies.

In addition to inconsistent results, earlier studies were mostly performed on western societies and little evidence is available from Asian societies, particularly from the Middle East where the prevalence of hypertension is estimated to be high (25\% of Iranian adults). ${ }^{13}$ To our knowledge, no study with large sample size is available in the Middle East to examine the association between dairy consumption and hypertension. Besides, dairy consumption among Middle Eastern populations is considerably lower than that in western societies. ${ }^{7,14}$

The low consumption of dairy products is more prevalent among university students than others because they usually adhere to unhealthy dietary behaviors. ${ }^{15}$ Dietary intakes among university students might be influenced by food prices, busy daily life, and preferences. ${ }^{16}$ University students are tended to consume fast foods, snacks, and sugar-sweetened beverages, rather than dairy products, fruits, and vegetables. ${ }^{17,18}$ In Spain, Alonso et al concluded that a higher intake of dairy products in university students was associated with reduced odds of hypertension. ${ }^{19}$ This association might be different among university students in the Middle East. Considering the aforesaid points, the current study was conducted to examine the association between dairy consumption and hypertension among a large population of university students in the Middle East.

\section{Materials and Methods \\ Participants}

The current research with a cross-sectional design was a part of the mental and physical health assessment of university student (MEPHASOUS) project which was performed in 2012-2013. This project was aimed to determine the contributing factors to the health problems and unhealthy behaviors of Iranian university students. Previously published studies presented details on the study design, sampling methods, and data gathering methods of the MEPHASOUS project. ${ }^{20,21}$ In the MEPHASOUS project, all students from 74 governmental eligible universities (in most provinces of Iran), related to the Ministry of Science and Technology (MST), were asked to take part in the study. Inclusion criteria were the registration in a governmental university and being in an age range of $\geq 18$ years. Both male and female students were included. All students were Iranian by nationality. No students from other countries of the Middle East were included. The collection of data was conducted in the health centers of universities. All required data on demographic variables, anthropometric indices, disease history, and dietary intakes were gathered from each student via pre-tested questionnaires. In total, 84,332 students participated in the current study; however, students with missing data on dairy consumption, blood pressure, and confounding variables were excluded $(n=17,321)$. This exclusion resulted in a dataset of 67,011 participants for the current analysis. We found no significant differences in terms of age, sex, and body mass index (BMI) between included and excluded students.

\section{Ethics}

The study was approved by the ethics committee of counseling and health organization of the Ministry of Science and Technology. All participants provided a signed informed consent document. Also, all procedures performed in this study were according to the ethical standards of the institutional and/or national research committee and with the 1964 Helsinki declaration and its later amendments or comparable ethical standards. 


\section{Dietary Assessment}

In order to collect information on dietary intake of selected food groups and also breakfast consumption, a validated 8 -item dietary habits questionnaire was used. This selfadministered questionnaire was filled out once by students. They were asked to report the frequency of consumption for fruits ( $<1 \mathrm{serv} /$ day, $1 \mathrm{serv} /$ day, $2-3 \mathrm{serv} /$ day, $\geq 4 \mathrm{serv} /$ day), vegetables ( $<1 \mathrm{serv} /$ day, $1 \mathrm{serv} / \mathrm{wk}, 2-3 \mathrm{serv} / \mathrm{wk}, \geq 4 \mathrm{serv} /$ wk), dairy products $(<1$ serv/day, 1 serv/wk, $2-3$ serv/wk, $\geq 4$ serv/wk), whole grains ( $<1$ serv/day, 1 serv/wk, $2-3$ serv/wk, $\geq 4 \mathrm{serv} / \mathrm{wk}$ ), fast foods (rarely, 1 time/wk, 2-3 times/wk, $\geq 4$ times/wk), sugar-sweetened beverages (SSBs) $(<1$ time/wk, 1-2 times/wk, $\geq 3$ times/wk), and sweets ( $<1 \mathrm{serv} / \mathrm{wk}, 1 \mathrm{serv} / \mathrm{wk}, 2-3 \mathrm{serv} / \mathrm{wk}, \geq 4 \mathrm{serv} / \mathrm{wk})$ over the last year and by considering the serving size of these foods. Students were instructed in the health centers of universities, prior to filling out the questionnaire, about the serving size of each food item. If needed, the standard serving sizes were shown to student using household measures. In the questionnaire, the response levels were different for each food according to its usual intake among the Iranian population. For example, the response categories for fruits which are frequently consumed by Iranians were in a daily format, while these categories for sweets that are infrequently consumed were in a weekly format. The last item of this questionnaire was about breakfast consumption. Students were asked to report how many times they consume breakfast in a week ( $<1$ day/wk, 1-2 days/wk, 3-4 days/wk, $\geq 5$ days/wk). Breakfast skipper was defined as individuals who consumed breakfast 4 days or less per week. A previously published article revealed the reliability and validity of the questionnaire. ${ }^{20}$ The questionnaire was specifically designed and validated for the MEPHASOUS project. ${ }^{20}$

In the current study, dairy products were considered as milk, cheese, yogurt and dough (yogurt drink). Participants were asked to consider all the products and then, reported their total consumption of dairy products. Indeed, data on individual dairy products were not collected. Students were asked not to consider the mixed foods that contain milk or other dairy products such as coffee/tea combined with milk, chocolate milk, and etc. These mixed foods usually contain a high amount of sugar and probably caffeine that may significantly affect the possible beneficial effect of dairy products of blood pressure. Other dairy-related products including butter and ice-cream which have little to no calcium were not considered in the assessment of dairy intake.
The reliability of the dietary habits questionnaire was examined in a separate study which was done on a subgroup of 70 students in each center of the MEPHASOUS project (total: 1960 students) ${ }^{20}$ In that study, a test-retest reliability process was applied in order to estimate the reliability of the questionnaire. Participants were asked to fill the dietary habits questionnaire two times with a 2-3 weeks' interval. The correlation coefficients for all dietary habits between the two times were more than 0.60 . Also, this coefficient for total dairy consumption was 0.72 indicating sufficient reliability of this questionnaire. The reliability and validity of the questionnaire were also confirmed by previous studies which applied this questionnaire for the assessment of dietary habits. ${ }^{21-23}$

\section{Definition of Hypertension}

Before the measurement of blood pressure, participants were asked to rest for $15 \mathrm{~min}$. Blood pressure was measured using a mercury sphygmomanometer (Model number: 58725-BK, Riester, Germany) two times with a 20-minute interval. During the measurement, participants were in a seated position. To decrease the measurement error, blood pressure was measured by one trained staff in each center of the MEPHASOUS project. The average of two measurements was considered as the final systolic and diastolic blood pressure. Hypertension was defined as systolic blood pressure (SBP) $\geq 140 \mathrm{mmHg}$ and diastolic blood pressure (DBP) $\geq 90 \mathrm{mmHg}$. This definition was based on the European Society of Cardiology (ESC)/European Society of Hypertension (ESH) guidelines in 2018. ${ }^{24}$

\section{Assessment of Other Variables}

We used a self-reported pre-tested questionnaire to collect data on age, sex (male/female), education [advanced diploma/bachelor of science (BSc)/master of science (MSc)/medical doctor (MD)/doctor of philosophy (Ph.D.)], marital status (single/married), occupation (having/not-having), health insurance (having/not-having), smoking (non-smoker/ex-smoker/current smoker), current use of nutritional supplements (including $\mathrm{Fe}, \mathrm{Ca}$, vitamins and other nutritional supplements) (yes/no), and family history of hypertension (yes/no) and diabetes (yes/no). Since health insurance in Iran can cost a lot for people, it was considered as an index for evaluation of economic status. Therefore, we considered students who had health insurance as economically "high" and those who did not have any type of health insurance as economically "low". In addition, sleep pattern was assessed by the two 
questions: "how is your pattern of sleeping and awaking?" and "how many hours do you sleep in a day?" The response options for the first question were "regular", "irregular" and for the second question were: " $<6$ hours/ day", "6-8 hours/day", "8-10 hours/day", ">10 hours/day. For physical activity assessment, students were asked how many times they exercise lasting $30 \mathrm{~min}$. They answered this question by these options: "rarely", "1-2 times/wk", "3-4 times/wk", ">5 times/wk". We considered students who conducted the exercise $\geq 3$ times/wk as physically active. Also, those who had the physical activity 1-2 times/wk were considered somewhat active and those who had a rare physical activity as inactive. In order to measure anthropometric indices, we employed a standard procedure to measure weight and height. We calculated BMI using the eligible formula. Those students with the BMI of $25-30$ and $\geq 30 \mathrm{~kg} / \mathrm{m}^{2}$ were considered as overweight or obese, respectively. ${ }^{25,26}$

\section{Statistical Analysis}

Participants were categorized according to the four levels of dairy consumption as follows: $<1$ serv/wk, 1 serv/wk, 2-3 serv/wk, $\geq 4$ serv/wk. We used the one-way analysis of variance (ANOVA) to assess differences in quantitative variables across the four levels of dairy consumption. To assess the distribution of categorical variables across dairy consumption levels, the Chi-square test was used. Binary logistic regression in adjusted models was employed to assess the association between dairy consumption and hypertension. In the first model, age and sex (not included in the sex-stratified analysis) were controlled. Further control was made for marital status, education, occupation, physical activity, economic status, smoking, sleep pattern, family history of hypertension (not included in the stratified analysis based on family history of hypertension) and diabetes, breakfast skipping, and supplement use in the second model. In the third model, additional adjustments were performed for dietary intakes of fruits, vegetables, fast foods, SSBs, whole grains, and sweets. In the final model, BMI (not included in the BMI status-stratified analysis) was additionally controlled to obtain an obesityindependent association between dairy consumption and hypertension. In these analyses, students in the first category of dairy consumption (who had the lowest consumption of dairy) were considered as reference. To obtain the overall trend of odds ratios across increasing categories of dairy consumption, we considered these categories as an ordinal variable in the logistic regression models. In addition to the whole population, we did subgroup analysis based on sex (male/female), BMI status $\left(<25 / \geq 25 \mathrm{~kg} / \mathrm{m}^{2}\right)$, and family history of hypertension (yes/no). All statistical analyses were done using SPSS software (version 19.0; SPSS Inc, Chicago IL). P values were considered significant at $<0.05$.

\section{Results}

The mean age of study participants was $21.4 \pm 3.9$ years and $55.5 \%$ were female subjects. In total, hypertension was prevalent among $6.9 \%$ of university students who participated in the current study. Out of 67,011 university students who participated in the current study, $23.2 \%$ were in the highest category of dairy consumption ( $\geq 4$ serv/wk) and $6.3 \%$ were in the lowest category ( $<1$ serv/wk). Also, $23.1 \%$ and $47.4 \%$ of students consumed 1 serv/wk and $2-3$ serv/wk dairy products, respectively.

Demographic characteristics and dietary habits of participants across categories of dairy consumption are shown in Table 1. Compared with individuals in the lowest category of dairy consumption, those in the highest category had higher age, weight, BMI, lower DBP, and were more likely to be female, married, physically active, obese, have a regular sleep pattern, use nutritional supplements, and less likely to be current smoker, economically low, breakfast skipper, and have family history of hypertension. In addition, the distribution of education and occupation was significantly different across categories of dairy consumption. Regards to dietary intakes, students in the top category of dairy consumption had greater intakes of fruits, vegetables, sweets, and lower intakes of fast foods, whole grains, and SSBs compared with those in the bottom category.

Multivariable odds ratios (ORs) and 95\% confidence intervals (CIs) for hypertension across different categories of dairy consumption are illustrated in Table 2. A significant inverse association was seen between dairy consumption and the odds of hypertension; such that after adjusting for potential confounders including demographic characteristics and dietary intakes, this association remained significant (OR: 0.16, 95\% CI: 0.13-0.19). Additional adjustment for BMI led to no alteration in the inverse association; such that individuals in the highest category of dairy consumption had $85 \%$ lower odds for having hypertension compared with those in the lowest category (OR: $0.15,95 \%$ CI: $0.13-0.18$ ).

\section{Findings from the Subgroup Analysis}

Multivariable ORs and 95\% CIs for hypertension across categories of dairy consumption stratified by sex, BMI 
Table I Demographic Characteristics and Dietary Habits of Participants Across Categories of Dairy Consumption ( $n=67,0 \mathrm{II})$

\begin{tabular}{|c|c|c|c|c|c|}
\hline & $<$ l serv/wk & I serv/wk & 2-3 serv/wk & $\geq 4$ serv/wk & P-trend* \\
\hline $\mathrm{N}(\%)$ & $4259(6.3)$ & $15,458(23.1)$ & $31,765(47.4)$ & $15,529(23.2)$ & \\
\hline Age (years) & $21.16 \pm 3.81$ & $21.27 \pm 3.70$ & $21.55 \pm 3.98$ & $21.47 \pm 4.19$ & $<0.001$ \\
\hline Sex (female) (\%) & 55.2 & 54.0 & 54.3 & 59.4 & $<0.001$ \\
\hline Weight (kg) & $63.64 \pm 13.96$ & $63.07 \pm 13.20$ & $64.22 \pm 13.99$ & $65.64 \pm 14.69$ & $<0.001$ \\
\hline BMI $\left(\mathrm{kg} / \mathrm{m}^{2}\right)$ & $22.39 \pm 3.93$ & $22.18 \pm 3.77$ & $22.47 \pm 3.98$ & $23.00 \pm 4.20$ & $<0.001$ \\
\hline Marital status (married) (\%) & 8.9 & 8.3 & 10.1 & 10.7 & $<0.001$ \\
\hline Education (graduated) (\%) & 34.2 & 36.0 & 39.7 & 34.8 & $<0.001$ \\
\hline Having a job (\%) & 11.6 & 8.8 & 9.6 & 10.8 & $<0.001$ \\
\hline Current smoker (\%) & 33.3 & 15.7 & 6.4 & 5.3 & $<0.001$ \\
\hline Physical activity (active) ${ }^{\mathrm{a}}(\%)$ & 36.7 & 34.6 & 39.2 & 45.6 & $<0.001$ \\
\hline Sleep pattern (regular) (\%) & 49.1 & 52.9 & 60.2 & 65.9 & $<0.001$ \\
\hline Economic status (low) (\%) & 29.1 & 22.2 & 16.8 & 13.1 & $<0.001$ \\
\hline Supplement use (\%) & 9.6 & 9.3 & 10.0 & 12.1 & $<0.001$ \\
\hline $\mathrm{DBP}(\mathrm{mmHg})$ & $78.84 \pm 19.90$ & $75.62 \pm 17.04$ & $74.34 \pm 15.28$ & $74.49 \pm 15.65$ & $<0.001$ \\
\hline $\mathrm{SBP}(\mathrm{mmHg})$ & $110.47 \pm 27.43$ & $107.54 \pm 20.98$ & $108.56 \pm 22.00$ & $108.38 \pm 25.39$ & 0.16 \\
\hline Family history of hypertension (\%) & 21.5 & 20.1 & 19.1 & 19.3 & $<0.001$ \\
\hline Family history of diabetes (\%) & 13.9 & 12.9 & 12.8 & 13.8 & 0.08 \\
\hline Overweight and obesity (\%) & 21.2 & 19.6 & 22.2 & 26.1 & $<0.001$ \\
\hline Breakfast skipping (\%) & 55.6 & 55.4 & 46.0 & 35.5 & $<0.001$ \\
\hline \multicolumn{6}{|l|}{ Dietary Intakes } \\
\hline Fruit ( $\geq 4$ serv/d) (\%) & 43.1 & 48.4 & 62.5 & 75.3 & $<0.001$ \\
\hline Vegetable ( $\geq 4$ serv/wk) (\%) & 11.3 & 7.2 & 11.5 & 24.1 & $<0.001$ \\
\hline Fast food ( $\geq 4$ time/wk) (\%) & 4.1 & 1.5 & 0.7 & 1.0 & $<0.001$ \\
\hline SSBs ( $\geq 3$ time/wk) (\%) & 10.9 & 5.5 & 5.3 & 7.9 & $<0.001$ \\
\hline Sweets ( $\geq 4$ serv/wk) (\%) & 14.7 & 10.1 & 11.7 & 16.9 & $<0.001$ \\
\hline Whole grains ( $\geq 4$ serv/wk) (\%) & 3.2 & 1.8 & 1.4 & 2.1 & $<0.001$ \\
\hline
\end{tabular}

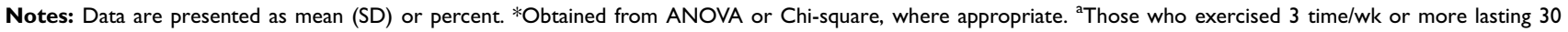
minutes in each time.

Abbreviations: BMI, body mass index; SSBs, sugar-sweetened beverages.

status and family history of hypertension are presented in Table 3. Before and after taking potential confounders into account, a significant inverse association was seen between dairy consumption and the odds of hypertension in the all subgroups including male (OR: 0.14, 95\% CI: $0.11-0.18$ ) and female (OR: 0.16 , 95\% CI: 0.12-0.21) individuals, normal-weight subjects (OR: 0.15, 95\% CI: $0.12-0.18$ ) and those with overweight or obesity (OR: 0.15, 95\% CI: 0.11-0.21), and individuals with (OR: 0.13, 95\% CI: 0.11-0.16) and without (OR: 0.24, 95\% CI: $0.17-0.35)$ a family history of hypertension.

\section{Discussion}

In the current study, we found that dairy consumption was inversely associated with the odds of hypertension. This association was significant even after controlling for potential confounding variables. Such a significant inverse association was also observed among males, females, normal-weight individuals, those with overweight or obesity and participants with and without a family history of hypertension. To the best of our knowledge, the current study is the first to investigate the association between dairy consumption and hypertension among university students in the Middle East.

The prevalence of hypertension has been increasing at an alarming rate. ${ }^{23,27}$ Different approaches including diet modifications have been introduced for preventing and controlling cardiovascular diseases such as hypertension. ${ }^{22,28-30}$ Dairy products contain minerals and bioactive compounds with antihypertensive properties. ${ }^{6,7}$ However, discrepant findings are available on the association between dairy consumption and hypertension. In the current study, we found a significant inverse association between dairy consumption and the odds of hypertension. Our findings were comparable with the study of Alonso et al, in which 5880 university graduates, aged $>20$ years, were followed for 2 years and the authors found that the hazard ratio (HR) of hypertension for extreme quintiles of low-fat dairy product consumption 
Table 2 Odds Ratios and 95\% Cls for Hypertension Across Categories of Dairy Consumption Among Whole Population ( $\mathrm{n}=67,0 \mathrm{I}$ )

\begin{tabular}{|l|l|l|l|l|l|}
\hline & < serv/wk & I serv/wk & 2-3 serv/wk & $\geq \mathbf{4}$ serv/wk & P-trend* \\
\hline $\mathrm{N}(\%)$ & $4259(6.3)$ & $15,458(23.1)$ & $31,765(47.4)$ & $15,529(23.2)$ \\
Crude & 1.00 & $0.32(0.29-0.34)$ & $0.10(0.09-0.11)$ & $0.08(0.07-0.09)$ & $<0.001$ \\
Model I & 1.00 & $0.32(0.29-0.34)$ & $0.10(0.09-0.11)$ & $0.08(0.07-0.09)$ & $<0.001$ \\
Model 2 & 1.00 & $0.49(0.43-0.56)$ & $0.24(0.21-0.28)$ & $0.18(0.15-0.21)$ & $<0.001$ \\
Model 3 & 1.00 & $0.47(0.41-0.54)$ & $0.22(0.19-0.26)$ & $0.16(0.13-0.19)$ & $<0.001$ \\
Model 4 & 1.00 & $0.47(0.41-0.55)$ & $0.22(0.19-0.26)$ & $0.15(0.13-0.18)$ & $<0.001$ \\
\hline
\end{tabular}

Notes: Data are presented as OR $(95 \% \mathrm{Cl})$. *Obtained from binary logistic regression. Model I: adjusted for age and sex. Model 2: additionally adjusted for marital status, education, occupation, physical activity, economic status, smoking, sleep pattern, family history of hypertension and diabetes, breakfast skipping, and supplement use. Model 3: further adjustment for dietary intake of fruits, vegetables, fast foods, sugar-sweetened beverages, whole grains, and sweets. Model 4: additionally control for BMI.

was 0.46 (95\% CI: $0.26,0.84)$ after adjustment for the main known risk factors for hypertension and several dietary factors. In that study, no significant association between whole-fat dairy products or total calcium intake and incident hypertension was reported. ${ }^{19}$ Also, in the study of Steffen et al which was done on 4304 participants aged 18-30 years at baseline and followed them for 15 years, intake of dairy desserts was associated with $28 \%$ lower risk of hypertension (HR: $0.74,95 \%$ CI: $0.60,0.92) .{ }^{31}$ In the Rotterdam study which was performed on 2245 adults, after 2 years follow-up, HRs of hypertension were 0.76 (95\% CI: $0.61,0.95)$ and 0.69 (95\% CI: $0.56,0.86$ ) for the highest versus lowest quartile of total dairy and low-fat dairy consumption, respectively. ${ }^{32}$ However, these risk reductions became non-significant after 6 years follow-up. In another prospective cohort study, Weng et al reported that dairy product consumption in combination with nuts decreased the incidence of hypertension. ${ }^{33}$ In the study of Buendia et al who combined three datasets of Nurses' Health Study (NHS), NHS II, and Health Professionals Follow-Up Study (HPFS), higher total dairy intake, especially in the form of yogurt, was associated with a lower risk of incident hypertension in middle-aged and older adult men and women. ${ }^{34}$ In addition to the preventive effects of dairy consumption on the incidence of hypertension, it may have a benefit for patients with hypertension. Lana et al reported that moderate consumption of low-fat milk/yogurt was associated with lower 24-hour diastolic blood pressure and better blood pressure control among older adults with hypertension. ${ }^{6}$ Contrary to our findings, Heraclides et al reported that intake of total dairy or specific dairy products was not associated with blood pressure and incident hypertension among a representative sample of British adults after 10 years follow-up. ${ }^{35}$ The observed discrepancies might be due to different processing and cooking methods of dairy products in different nations which may affect the quality of dairy products. Moreover, the lack of adjustment for important confounders might be another reason for the discrepancy. For example, in the study of Heraclides et al in which dairy consumption was not significantly associated with hypertension, some potential confounders including age, sex, family history of hypertension, and use of nutritional supplements were not controlled, while in the current study, we took the confounders into account.

In the current study, despite the higher prevalence of obesity in the highest versus lowest category of dairy consumption, the odds of hypertension was reduced in this comparison. Obesity is a known risk factor for hypertension. ${ }^{36}$ Although the confounding effect of obesity was adjusted in the current analysis, the observed disparity might be explained by other factors related to hypertension. For instance, students in the highest category of dairy consumption, despite the obesity, had greater intakes of fruits and vegetables, lower intakes of fast foods and SSBs, and were more likely to be physically active and non-smokers compared with those in the lowest category. Therefore, the beneficial effects of these factors can alleviate the adverse effect of obesity on blood pressure. , 5,23

University students usually have different dietary behaviors compared with others. ${ }^{17,18}$ Food choices of this population might be affected by several factors including food prices, busy daily life, and preferences. ${ }^{16}$ Therefore, students prefer to consume cheap or ready-to-eat foods such as fast foods, snacks, and sweets. ${ }^{18,37}$ However, due to the moderate-to-high cost of dairy products in Iran, the consumption of these products is estimated to be low among university students. ${ }^{7,14}$ As seen in the current study, only $23.2 \%$ of students consumed $\geq 4$ serv/wk dairy products (Table 1). These students were defined as high dairy consumers in this study. Although they had low consumption of dairy products $(\geq 4$ serv/wk) compared with high dairy consumers ( $>3$ serv/day) in studies performed on Western populations, ${ }^{31}$ this low amount also 
Table 3 Odds Ratios and 95\% Cls for Hypertension Across Categories of Dairy Consumption Stratified by Sex, BMI Status and Family History of Hypertension

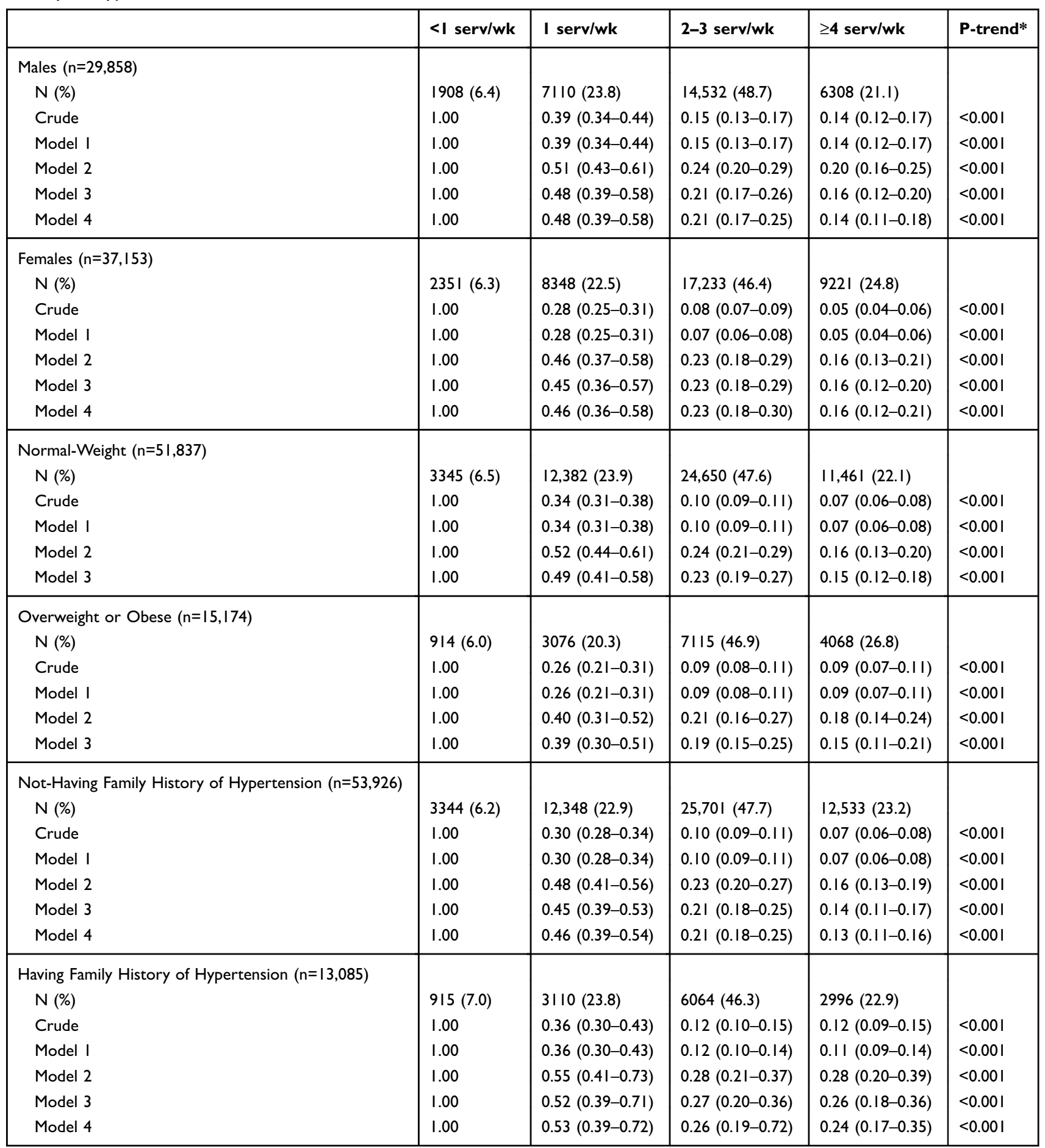

Notes: Data are presented as OR $(95 \% \mathrm{Cl})$. $*$ Obtained from binary logistic regression, Model I: adjusted for age and sex (not included in the sex-stratified analysis). Model 2: additionally adjusted for marital status, education, occupation, physical activity, economic status, smoking, sleep pattern, family history of hypertension (not included in the stratified analysis based on family history of hypertension) and diabetes, breakfast skipping and supplement use. Model 3: further adjustment for dietary intake of fruits, vegetables, fast foods, sugar-sweetened beverages, whole grains, and sweets and desserts. Model 4: additionally control for BMI (not included in the BMI status-stratified analysis).

provides the protective association between dairy consumption and hypertension.

In the current study, we could not determine the type of hypertension (primary and secondary) due to the lack of required data. The etiology of primary hypertension is unknown, but secondary hypertension is due to an identifiable cause. ${ }^{38}$ Since secondary hypertension occurs in early ages, ${ }^{38}$ most cases of hypertension in the current study 
may be secondary. However, it has been shown that the prevalence of primary hypertension among children, adolescences, and young adults is increasing at an alarming rate. ${ }^{39}$ For instance, recent reports from the US population demonstrated that primary hypertension is prevalent among $>10 \%$ of children and adolescences which rises by increasing age. ${ }^{39}$ This increment is due to the obesity epidemic, unhealthy dietary behaviors, and sedentary lifestyle in these age groups. ${ }^{40}$ Overall, it can be concluded that both cases of primary and secondary hypertension were present in the current study that both are affected by dietary factors.

Several mechanisms have been proposed for the inverse association between dairy consumption and hypertension. Dairy products are rich sources of potassium, magnesium, phosphorus, and vitamin $\mathrm{D},{ }^{11}$ all of which are involved in the BP-lowering effects of dairy products such as regulation of vascular resistance and promotion of vasodilation by enhancing the synthesis of nitric oxide, reducing renal sodium retention, blocking calcium channels and reducing intracellular calcium. ${ }^{41-45}$ Moreover, bioactive peptides that are released during dairy protein digestion have an inhibitory effect on ACE resulting in reduced blood levels of angiotensin, preventing blood vessel constriction, and modulating endothelial function. ${ }^{46,47}$ Furthermore, specific peptides present in dairy products may reduce the secretion of endothelin-1 by endothelial cells. ${ }^{48,49}$ Endothelins are proteins that contribute to the constriction of blood vessels, thereby raising blood pressure. ${ }^{48}$

This study has several strengths. A wide range of confounders including socio-demographic variables, dietary intakes of selected food groups and BMI were controlled in the analyses. In addition to the whole population, we performed stratified analyses based on sex, BMI status and family history of hypertension. Despite these strengths, some limitations should be taken into account for interpreting our findings. Since the design of our study was crosssectional, we could not confer a causal association between dairy consumption and the odds of hypertension. Due to the large sample size of the MEPHASOUS project and in order to fill the questionnaire in minimum time, we had to apply a short form of dietary habits questionnaire which contained the foods that are usually consumed by students. Therefore, no items for individual dairy products and their quality (such as low/high-fat dairy) were not present in that questionnaire. Hence, in the current study, we were unable to assess the association of individual dairy products and their quality with hypertension. Besides, due to the lack of data for all food items that students usually consume, we could not calculate total energy intake. Therefore, adjustment for total energy intake that is an important confounder in diet-disease relationships was impossible in the current study. However, we controlled for intakes of all food groups that were assessed in the current study to diminish the confounding effect of energy intake. Also, given that BMI is positively associated with total energy intake, adjustment for this variable could reduce the confounding effect of energy intake on the association between dairy consumption and hypertension. In addition, we did not adjust for dietary intake of sodium which has a major contribution to hypertension. ${ }^{50}$ However, intake of some sodium-rich food groups such as fast foods was controlled in the analysis. Fast foods are the most commonly used snacks among Iranian adults. ${ }^{51}$ However, we cannot exclude the effects of other sodium-containing foods such as salt and salty nuts on the association between dairy consumption and hypertension. Unfortunately, we did not collect data on intakes of these foods in the MEPHASOUS project. Therefore, our findings should be considered with caution. In the current study, only health insurance (having/not-having) was considered as an index for evaluation of economic status. We had no information on other factors related to economic status such as house possession or having a car to evaluate economic status more accurately; however, based on previous studies, health insurance is an important indicator of economic well-being around the world. ${ }^{52,53}$ Although a validated questionnaire was applied to assess dietary intakes in our study, the closed-end response nature of the questionnaire might increase the rate of misclassification. There is a 7-8 year gap between data collection and analysis in the current study. Despite the big gap, we think that our results reflect the present hypertension scenario because they were in line with the findings of several studies that examined the association between dairy consumption and hypertension. The presence of recall bias in this study, like other questionnaire-based cross-sectional studies, is unavoidable. However, since the study participants were high-educated, this bias in the present study may be lower than other studies that were performed on low-educated participants.

In conclusion, we found that dairy consumption was inversely associated with odds of hypertension. Such an inverse association was also found in males, females, normal-weight individuals, those with overweight or obesity and participants with and without a family history of hypertension. Further studies, particularly randomized clinical trials, are needed to determine the effects of 
dairy consumption on blood pressure in healthy individuals and those with elevated blood pressure.

\section{Acknowledgment}

This work conducted based on MEPHASOUS (Mental and Physical Health Assessment of University Student) study which was designed by the Counseling and Health Organization of the Ministry of Science and Technology (CHOMST), Tehran, Iran. We are very thankful to numerous colleagues with whom we have shared our research on dairy consumption and its effects on blood pressure in large population students and who have helped us with valuable comments.

\section{Disclosure}

The authors report no conflicts of interest in this work.

\section{References}

1. Giles TD, Materson BJ, Cohn JN, Kostis JB. Definition and classification of hypertension: an update. J Clin Hypertens (Greenwich). 2009;11(11):611-614. doi:10.1111/j.1751-7176.2009.00179.x

2. Lim SS, Vos T, Flaxman AD, et al. A comparative risk assessment of burden of disease and injury attributable to 67 risk factors and risk factor clusters in 21 regions, 1990-2010: a systematic analysis for the global burden of disease study 2010. Lancet. 2012;380 (9859):2224-2260. doi:10.1016/S0140-6736(12)61766-8

3. Kearney PM, Whelton M, Reynolds K, Muntner P, Whelton PK, He J. Global burden of hypertension: analysis of worldwide data. Lancet 2005;365(9455):217-223. doi:10.1016/S0140-6736(05)17741-1

4. Williams B, Mancia G, Spiering W, Rosei EA, Azizi M, Burnier M. 2018 practice guidelines for the management of arterial hypertension of the European society of hypertension and the european society of cardiology: $\mathrm{ESH} / \mathrm{ESC}$ task force for the management of arterial hypertension: erratum. J Hypertens. 2019;37(2):456. doi:10.1097/HJH.0000000000002026

5. Leung AA, Nerenberg K, Daskalopoulou SS, et al. Hypertension Canada's 2016 Canadian hypertension education program guidelines for blood pressure measurement, diagnosis, assessment of risk, prevention, and treatment of hypertension. Can J Cardiol. 2016;32 (5):569-588. doi:10.1016/j.cjca.2016.02.066

6. Lana A, Banegas JR, Guallar-Castillon P, Rodriguez-Artalejo F, Lopez-Garcia E. Association of dairy consumption and 24-hour blood pressure in older adults with hypertension. Am J Med. 2018;131(10):1238-1249. doi:10.1016/j.amjmed.2018.04.039

7. Sadeghi O, Keshteli AH, Doostan F, Esmaillzadeh A, Adibi P. Association between dairy consumption, dietary calcium intake and general and abdominal obesity among Iranian adults. Diabetes Metab Syndr. 2018;12(5):769-775. doi:10.1016/j.dsx.2018.04.040

8. Majumder $\mathrm{K}, \mathrm{Wu} \mathrm{J}$. Molecular targets of antihypertensive peptides: understanding the mechanisms of action based on the pathophysiology of hypertension. Int J Mol Sci. 2014;16(1):256-283. doi:10.3390/ ijms16010256

9. Fekete AA, Givens DI, Lovegrove JA. Casein-derived lactotripeptides reduce systolic and diastolic blood pressure in a meta-analysis of randomised clinical trials. Nutrients. 2015;7(1):659-681. doi:10.3390/nu7010659

10. Cicero AF, Aubin F, Azais-Braesco V, Borghi C. Do the lactotripeptides isoleucine-proline-proline and valine-proline-proline reduce systolic blood pressure in European subjects? A meta-analysis of randomized controlled trials. Am J Hypertens. 2013;26(3):442-449. doi:10.1093/ajh/hps044
11. Wang H, Fox CS, Troy LM, McKeown NM, Jacques PF. Longitudinal association of dairy consumption with the changes in blood pressure and the risk of incident hypertension: the Framingham Heart Study. Br J Nutr. 2015;114(11):1887-1899. doi:10.1017/ S0007114515003578

12. Ding M, Huang T, Bergholdt HK, Nordestgaard BG, Ellervik C, Qi L. Dairy consumption, systolic blood pressure, and risk of hypertension: mendelian randomization study. BMJ. 2017;356:j1000. doi:10.1136/bmj.j1000

13. Esteghamati A, Abbasi M, Alikhani S, et al. Prevalence, awareness, treatment, and risk factors associated with hypertension in the Iranian population: the national survey of risk factors for noncommunicable diseases of Iran. Am J Hypertens. 2008;21(6):620-626. doi:10.1038/ ajh.2008.154

14. Abdi F, Atarodi KZ, Mirmiran P, Esteki T. Surveying Global and Iranian Food Consumption Patterns: a Review of the Literature. 2015:159-167.

15. Mahon AK, Haas EJ. A mixed-methods approach to targeting college students' dairy behaviors. Am J Health Behav. 2013;37(5):703-710. doi:10.5993/AJHB.37.5.14

16. Vilaro MJ, Colby SE, Riggsbee K, et al. Food choice priorities change over time and predict dietary intake at the end of the first year of college among students in the U.S. Nutrients. 2018;10 (9):1296. doi:10.3390/nu10091296

17. Hilger J, Loerbroks A, Diehl K. Eating behaviour of university students in Germany: dietary intake, barriers to healthy eating and changes in eating behaviour since the time of matriculation. Appetite. 2017;109:100-107. doi:10.1016/j.appet.2016.11.016

18. Betancourt-Nunez A, Marquez-Sandoval F, Gonzalez-Zapata LI, Babio N, Vizmanos B. Unhealthy dietary patterns among healthcare professionals and students in Mexico. BMC Public Health. 2018;18 (1):1246. doi:10.1186/s12889-018-6153-7

19. Alonso A, Beunza JJ, Delgado-Rodriguez M, Martinez JA, MartinezGonzalez MA. Low-fat dairy consumption and reduced risk of hypertension: the Seguimiento Universidad de Navarra (SUN) cohort. Am J Clin Nutr. 2005;82(5):972-979. doi:10.1093/ajcn/82.5.972

20. Mansouri M, Sharifi F, Varmaghani M, et al. Iranian university students lifestyle and health status survey: study profile. J Diabetes Metab Disord. 2017;16(1):48. doi:10.1186/s40200-017-0329-z

21. Mansouri M, Hasani-Ranjbar S, Yaghubi H, et al. Breakfast consumption pattern and its association with overweight and obesity among university students: a population-based study. Eat Weight Disord. 2018:1-9.

22. Mansouri M, Miri A, Varmaghani M, et al. Vitamin D deficiency in relation to general and abdominal obesity among high educated adults. Eat Weight Disord. 2019;24(1):83-90. doi:10.1007/s40519-018-0511-4

23. Mansouri M, Sharifi F, Yaghubi H, et al. Sugar-sweetened beverages consumption in relation to hypertension among Iranian university students: the MEPHASOUS study. Eat Weight Disord. 2019. doi:10.1007/s40519-019-00713-9

24. Williams B, Mancia G, Spiering W, et al. 2018 ESC/ESH guidelines for the management of arterial hypertension: the task force for the management of arterial hypertension of the European Society of Cardiology (ESC) and the European Society of Hypertension (ESH). Eur Heart J. 2018;39:3021-3104.

25. Dabbagh-Moghadam A, Mozaffari-Khosravi H, Nasiri M, Miri A, Rahdar M, Sadeghi O. Association of white and red meat consumption with general and abdominal obesity: a cross-sectional study among a population of Iranian military families in 2016. Eat Weight Disord. 2017;22(4):717-724. doi:10.1007/s40519-017-0385-x

26. Miri A, Nasiri M, Zonoori S, et al. The association between obesity and migraine in a population of Iranian adults: a case-control study. Diabetes Metab Syndr. 2018;12(5):733-736. doi:10.1016/j.dsx.2018.04.020

27. Parohan M, Sadeghi A, Nasiri M, et al. Dietary acid load and risk of hypertension: a systematic review and dose-response meta-analysis of observational studies. Nutr Metab Cardiovasc Dis. 2019;29 (7):665-675. doi:10.1016/j.numecd.2019.03.009 
28. Pahlavani N, Jafari M, Sadeghi O, et al. L-arginine supplementation and risk factors of cardiovascular diseases in healthy men: a double-blind randomized clinical trial. F1000Res. 2014;3:306. doi:10.12688/f1000research.5877.1

29. Mansouri M, Abasi R, Nasiri M, et al. Association of vitamin D status with metabolic syndrome and its components: a cross-sectional study in a population of high educated Iranian adults. Diabetes Metab Syndr. 2018;12(3):393-398. doi:10.1016/j.dsx.2018.01.007

30. Ghorabi S, Salari-Moghaddam A, Daneshzad E, Sadeghi O, Azadbakht L, Djafarian K. Association between the DASH diet and metabolic syndrome components in Iranian adults. Diabetes Metab Syndr. 2019;13(3):1699-1704. doi:10.1016/j.dsx.2019.03.039

31. Steffen LM, Kroenke CH, Yu X, et al. Associations of plant food, dairy product, and meat intakes with 15-y incidence of elevated blood pressure in young black and white adults: the coronary artery risk development in young adults (CARDIA) study. Am J Clin Nutr. 2005;82(6):1169-1177. doi:10.1093/ajen/82.6.1169

32. Engberink MF, Hendriksen MA, Schouten EG, et al. Inverse association between dairy intake and hypertension: the rotterdam study. $\mathrm{Am}$ J Clin Nutr. 2009;89(6):1877-1883. doi:10.3945/ajen.2008.27064

33. Weng LC, Steffen LM, Szklo M, Nettleton J, Chambless L, Folsom AR. A diet pattern with more dairy and nuts, but less meat is related to lower risk of developing hypertension in middle-aged adults: the Atherosclerosis Risk in Communities (ARIC) study. Nutrients. 2013;5(5):1719-1733. doi:10.3390/nu5051719

34. Buendia JR, Li Y, Hu FB, et al. Long-term yogurt consumption and risk of incident hypertension in adults. J Hypertens. 2018;36 (8):1671-1679. doi:10.1097/HJH.0000000000001737

35. Heraclides A, Mishra GD, Hardy RJ, et al. Dairy intake, blood pressure and incident hypertension in a general British population: the 1946 birth cohort. Eur J Nutr. 2012;51(5):583-591. doi:10.1007/ s00394-011-0242-z

36. Lavie CJ, De Schutter A, Parto P, et al. Obesity and prevalence of cardiovascular diseases and prognosis-the obesity paradox updated. Prog Cardiovasc Dis. 2016;58(5):537-547. doi:10.1016/j.pcad.2016. 01.008

37. Mohammadbeigi A, Asgarian A, Moshir E, et al. Fast food consumption and overweight/obesity prevalence in students and its association with general and abdominal obesity. J Prev Med Hyg. 2018;59(3): E236-e40. doi:10.15167/2421-4248/jpmh2018.59.3.830

38. Rimoldi SF, Scherrer U, Messerli FH. Secondary arterial hypertension: when, who, and how to screen? Eur Heart J. 2014;35 (19):1245-1254. doi:10.1093/eurheartj/eht534

39. Falkner B. The childhood role in development of primary hypertension. Am J Hypertens. 2018;31(7):762-769. doi:10.1093/ ajh/hpy058

40. Anyaegbu EI, Dharnidharka VR. Hypertension in the teenager. Pediatr Clin North Am. 2014;61(1):131-151. doi:10.1016/j.pcl.2013. 09.011
41. Appel LJ, Brands MW, Daniels SR, Karanja N, Elmer PJ, Sacks FM. Dietary approaches to prevent and treat hypertension: a scientific statement from the American heart association. Hypertension. 2006;47(2):296-308. doi:10.1161/01.HYP.0000202568.01167.B6

42. McGrane MM, Essery E, Obbagy J, et al. Dairy Consumption, blood pressure, and risk of hypertension: an evidence-based review of recent literature. Curr Cardiovasc Risk Rep. 2011;5(4):287-298. doi:10.1007/s12170-011-0181-5

43. Park KM, Cifelli CJ. Dairy and blood pressure: a fresh look at the evidence. Nutr Rev. 2013;71(3):149-157. doi:10.1111/nure.12017

44. Ralston RA, Lee JH, Truby H, Palermo CE, Walker KZ. A systematic review and meta-analysis of elevated blood pressure and consumption of dairy foods. J Hum Hypertens. 2012;26(1):3-13. doi:10.1038/ jhh.2011.3

45. Soedamah-Muthu SS, Verberne LD, Ding EL, Engberink MF, Geleijnse JM. Dairy consumption and incidence of hypertension: a dose-response meta-analysis of prospective cohort studies. Hypertension. 2012;60(5):1131-1137. doi:10.1161/HYPERTENSION AHA.112.195206

46. Boelsma E, Kloek J. Lactotripeptides and antihypertensive effects: a critical review. Br J Nutr. 2009;101(6):776-786. doi:10.1017/ S0007114508137722

47. Severin S, Wenshui X. Milk biologically active components as nutraceuticals: review. Crit Rev Food Sci Nutr. 2005;45(7-8):645-656. doi:10.1080/10408690490911756

48. Maes W, Van Camp J, Vermeirssen V, et al. Influence of the lactokinin Ala-Leu-Pro-Met-His-Ile-Arg (ALPMHIR) on the release of endothelin-1 by endothelial cells. Regul Pept. 2004;118(1-2):105-109. doi:10.1016/j.regpep.2003.11.005

49. Maki KC, Rains TM, Schild AL, et al. Effects of low-fat dairy intake on blood pressure, endothelial function, and lipoprotein lipids in subjects with prehypertension or stage 1 hypertension. Vasc Health Risk Manag. 2013;9:369-379. doi:10.2147/VHRM.S45684

50. Huang L, Trieu K, Yoshimura S, et al. Effect of dose and duration of reduction in dietary sodium on blood pressure levels: systematic review and meta-analysis of randomised trials. BMJ. 2020;368: m315. doi:10.1136/bmj.m315

51. Bahadoran Z, Mirmiran P, Golzarand M, Hosseini-Esfahani F, Azizi F. Fast food consumption in Iranian adults; dietary intake and cardiovascular risk factors: tehran Lipid and Glucose Study. Arch Iran Med. 2012;15(6):346-351.

52. Burkhauser RV, Simon KI. Measuring the Impact of Health Insurance on Levels and Trends in Inequality. National Bureau of Economic Research; 2010. Report No.: 0898-2937.

53. Bedard K, Deschenes O. Sex preferences, marital dissolution, and the economic status of women. J Human Resources. 2005;40 (2):411-434. doi:10.3368/jhr.XL.2.411

Diabetes, Metabolic Syndrome and Obesity: Targets and Therapy

\section{Publish your work in this journal}

Diabetes, Metabolic Syndrome and Obesity: Targets and Therapy is an international, peer-reviewed open-access journal committed to the rapid publication of the latest laboratory and clinical findings in the fields of diabetes, metabolic syndrome and obesity research. Original research, review, case reports, hypothesis formation, expert opinion and commentaries are all considered for publication. The manuscript management system is completely online and includes a very quick and fair peer-review system, which is all easy to use. Visit http://www.dovepress.com/testimonials.php to read real quotes from published authors. 